\title{
Intramolecular Michael Reactions by Iron(III) Catalysis
}

\author{
Jens Christoffers* and Heiko Oertling \\ Technische Universität Berlin, Institut für Organische Chemie, Sekretariat C3, Straße des 17. Juni 135, D-10623 Berlin, Germany
}

Received 7 December 1999; accepted 10 January 2000

\begin{abstract}
Seven-membered ring annulation is achieved by an intramolecular Michael reaction catalyzed by iron(III) chloride hexahydrate. Reaction conditions are mild and non-basic, and chemo- and stereoselectivity is excellent. Contrastingly, this method fails for a 17-membered ring annulation. (C) 2000 Elsevier Science Ltd. All rights reserved.
\end{abstract}

\section{Introduction}

Iron(III) chloride hexahydrate is to date the most efficient catalyst for the Michael reaction of $\beta$-keto esters with vinyl ketones. ${ }^{1-3}$ Owing to the mild and non-basic reaction conditions, the chemoselectivity of this process is superior to that offered by base catalysis, ${ }^{4-8}$ since the latter suffers from various unwanted side- and subsequent reactions, such as aldol cyclizations, ester solvolysis or retro-Claisen type decompositions. Moreover, since conversions are quantitative within hours at room temperature, the isolated yields are generally very good. ${ }^{9}$ Inspired by these results on intermolecular Michael reactions we considered $\mathrm{FeCl}_{3} \cdot 6 \mathrm{H}_{2} \mathrm{O}$ to be also an efficient catalyst for intramolecular conversions, ${ }^{10-15}$ with special respect to macrocyclizations still being a challenging goal in synthetic organic chemistry. In the past some reports on macrocyclizations by intramolecular Michael reactions have been reported by others. ${ }^{16-19}$ Herein we wish to report on our results on seven- ${ }^{20}$ and seventeen-membered carboannulations by intramolecular Michael reactions catalyzed by $\mathrm{FeCl}_{3} \cdot 6 \mathrm{H}_{2} \mathrm{O}$.

\section{Results and Discussion}

\section{Seven-membered ring annulation}

Donor-acceptor functionalized precursor 1a was cleanly converted with a catalytic amount of $\mathrm{FeCl}_{3} \cdot 6 \mathrm{H}_{2} \mathrm{O}$ to the bicyclo[5.5.0]-dodecane derivative 2a (Scheme 1). The reaction proceeded at ambient temperature in $\mathrm{CH}_{2} \mathrm{Cl}_{2}$ as solvent within a few hours, and the isolated yield was good $(80 \%)$. Separation of the product from all ironcontaining materials was simply accomplished by filtration through $\mathrm{SiO}_{2}$. Basic reaction conditions were avoided, thus, no side- or subsequent products were observed. Moreover, no dimeric or oligomeric species resulting from an intermolecular process were detectable by GC-MS. Besides a very high chemoselectivity, quantitative stereoselectivity was observed: only one diastereoisomer of the racemic material 2a was isolated. After assignment of all proton and carbon NMR resonances by H,H-COSY, DEPT, HMBC and HMQC experiments the relative configuration was established to be trans: A $J$-resolved $500 \mathrm{MHz}{ }^{1} \mathrm{H}$ NMR<smiles>C=CC(=O)CCC1CCCCC(=O)C1C(C)=O</smiles>

1a, $\mathrm{n}=1$

1b, $n=11$

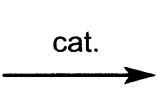

2a, $\mathrm{n}=1,80 \%$

$2 \mathbf{b}, \mathrm{n}=11,8 \%$

Scheme 1. Iron(III) chloride catalyzed intramolecular Michael reactions; reagents and conditions: $1 \mathrm{a}, 5 \mathrm{~mol} \% \mathrm{FeCl}_{3} \cdot 6 \mathrm{H}_{2} \mathrm{O}, \mathrm{CH}_{2} \mathrm{Cl}_{2}, 23^{\circ} \mathrm{C}, 12 \mathrm{~h}, 80 \%$ yield of 2a, $11 \%$ of $1 \mathbf{a}$ was recovered; $\mathbf{1 b}, 10 \mathrm{~mol} \% \mathrm{FeCl}_{3}, \mathrm{CH}_{2} \mathrm{Cl}_{2}, 2 \mathrm{~h}$ ultrasonication at $23^{\circ} \mathrm{C}$, then $6 \mathrm{~h}$ stirring at $23^{\circ} \mathrm{C}$.

Keywords: annulation; catalysis; iron compounds; Michael reactions.

* Corresponding author. Tel.: +49-30/314-23189; fax: +49-30/723-1233; e-mail: jchr@wap0105.chem.tu-berlin.de 


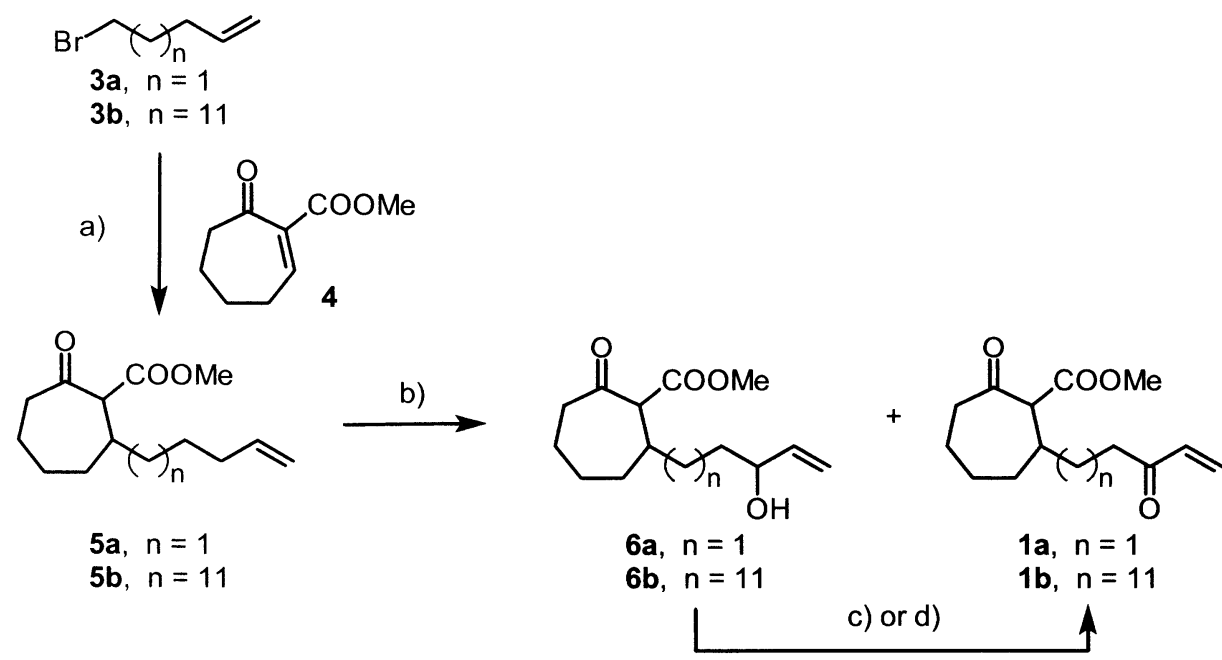

Scheme 2. Reagents, conditions, and yields: (a) $1 . \mathrm{Mg}$, THF (ultrasound for $\mathbf{3 b}$ ), $2 . \mathrm{CuI},-20^{\circ} \mathrm{C}, 1 \mathrm{~h}, 3.4$ (0.7 equiv.), $-78^{\circ} \mathrm{C}$ to $23^{\circ} \mathrm{C}, \mathbf{5 a}: 85 \%, \mathbf{5 b}: 36 \%$; (b) $\mathrm{SeO}_{2}$ (2 equiv.), $t \mathrm{BuO}_{2} \mathrm{H}$ (5 equiv.), $\mathrm{CH}_{2} \mathrm{Cl}_{2}, 23^{\circ} \mathrm{C}, 15 \mathrm{~h}, \mathbf{6 a}: 38 \%$ (1a: $30 \%, 5 \mathbf{a}: 27 \%$ recovered), $6 \mathbf{b}: 60 \%$; (c) for $6 \mathbf{a}: 1 . \mathrm{PCC}\left(1.5\right.$ equiv.), $\mathrm{CH}_{2} \mathrm{Cl}{ }_{2}, 23^{\circ} \mathrm{C}, 4$ h, 2 .

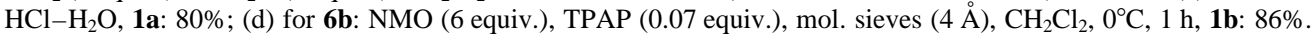

spectrum gave a triplet-triplet coupling pattern of the 7-H (methine) signal at $1.71 \mathrm{ppm}$, representing a strictly symmetrical environment of this proton with two equatorial $(J=3 \mathrm{~Hz})$ and two axial $(J=11 \mathrm{~Hz})$ vicinal protons. Such a high symmetry can only be achieved with trans-connection of the two seven-membered rings.

\section{Seventeen-membered ring annulation}

Encouraged by the latter result we converted the starting material $\mathbf{1 b}$ bearing a $\mathrm{C}_{15}$-side chain with a catalytic amount of $\mathrm{FeCl}_{3} \cdot 6 \mathrm{H}_{2} \mathrm{O}$ under both high-dilution and pseudo-highdilution conditions. ${ }^{21}$ To our surprise we found conversion of $\mathbf{1 b}$ to products with either water or $\mathrm{MeOH}$ (from the $\mathrm{CH}_{2} \mathrm{Cl}_{2}$ solvent applied) being added to the vinyl ketone moiety. Consequently, in further experiments we excluded moisture, applied anhydrous $\mathrm{FeCl}_{3}$ and used $\mathrm{MeOH}$-free $\mathrm{CH}_{2} \mathrm{Cl}_{2}$ as solvent.

However, at ambient temperature under pseudo-highdilution conditions (addition of a solution of substrate $\mathbf{1 b}$ to up to 10 equiv. of a $10 \mathrm{~g} / 1$ solution of $\mathrm{FeCl}_{3}$ in $\mathrm{CH}_{2} \mathrm{Cl}_{2}$ over a period up to 5 days) only starting material was recovered almost quantitatively. Thus, we decided to run the reaction at elevated temperature (up to $70^{\circ} \mathrm{C}$ in a sealed flask) under diluted conditions (1.5-25 mmol/1 in THF or $\mathrm{CH}_{2} \mathrm{Cl}_{2}$ with $0.1-1$ equiv. $\mathrm{FeCl}_{3}$ ), but again only starting material was recoverable after workup in 14-40\% yield, and no other unique material was identified after chromatography. It is important to note, that we were neither able to accomplish the conversion of $\mathbf{1 b}$ to $\mathbf{2} \mathbf{b}$ with a basic catalyst $\left(\mathrm{Cs}_{2} \mathrm{CO}_{3}\right.$ in $\left.\mathrm{THF}\right)$.

Finally, compound $\mathbf{1 b}$ was converted with 0.1 equiv. $\mathrm{FeCl}_{3}$ in $100 \mathrm{mmol} / 1 \mathrm{CH}_{2} \mathrm{Cl}_{2}$. Immediate precipitation of a irondionato complex occurred, but after $2 \mathrm{~h}$ of ultrasonication at ambient temperature a homogeneous mixture was obtained, which was further stirred for $6 \mathrm{~h}$. After workup and twice chromatography about $8 \%$ of product $\mathbf{2 b}$ was isolated besides various presumably dimeric or oligomeric species (Scheme 1). Characterization of $\mathbf{2 b}$ is based on HRMS and
${ }^{1} \mathrm{H}$ NMR spectroscopy. The latter shows no more olefinic signals, but four distinguished $\mathrm{MeO}$ resonances in equal ratio at 3.70 to $3.75 \mathrm{ppm}$ being due to the existence of two relatively stable conformers each of two diastereoisomers.

\section{Syntheses of precursors}

Syntheses of donor-acceptor functionalized substrates $\mathbf{1 a}, \mathbf{b}$ started with $\omega$-bromo- $\alpha$-olefins $\mathbf{3 a , b}$ being converted after Grignard formation (ultrasonication was required in case of 3b) and transmetallation with $\mathrm{CuI}^{22}$ with the 2-acceptor substituted cycloalkenone $\mathbf{4}$ to yield cyclic oxoesters $\mathbf{5 a}, \mathbf{b}$ with an $\omega$-alkenyl side chain. In case of $\mathbf{5 b}$ the low yield (36\%) might be due to the tautomerization of $\mathbf{4}$ to the corresponding dienol, ${ }^{23}$ which was isolated in $34 \%$ yield as a byproduct and could protonate the cuprate derived from 3b under reaction conditions.

Subsequently, compounds 5a,b were submitted to allylic oxidation with freshly sublimated $\mathrm{SeO}_{2}$ and $t \mathrm{BuO}_{2} \mathrm{H}^{24-26}$ In case of $5 \mathbf{a}$ besides the product $6 \mathbf{a}(38 \%)$, enone 1a (30\%) was formed and starting material 5a was recovered (27\%). All three compounds were separable by column chromatography. In case of $\mathbf{5 b}$ allylic alcohol $\mathbf{6 b}$ was obtained in $60 \%$ yield. In both cases increase of reaction times or temperatures did not raise yields, but lead to unspecified decomposition making the purification of products more difficult.

Allylic alcohol 6a was oxidized to the enone $1 \mathbf{a}$ by $\mathrm{PCC}^{27}$ in $80 \%$ yield, compound $\mathbf{6 b}$ was oxidized by the system TPAP-NMO ${ }^{28}$ to give $\mathbf{1 b}$ in $86 \%$ yield (Scheme 2 ).

Whereas $\omega$-bromo- $\alpha$-olefin $3 \mathbf{a}$ was commercially available, compound 3b was not and had to be prepared by copper cross-coupling reaction ${ }^{29}$ of a $\alpha, \omega$-dibromo alkane 7 with an allyl Grignard reagent (Scheme 3). An optimal yield of $47 \%$ of $\mathbf{3 b}$ was obtained and $\alpha, \omega$-diolefin $\mathbf{8}$ was isolated as the byproduct in $12 \%$ yield. The separation of $7, \mathbf{3 b}$ and $\mathbf{8}$ was accomplished by column chromatography. 


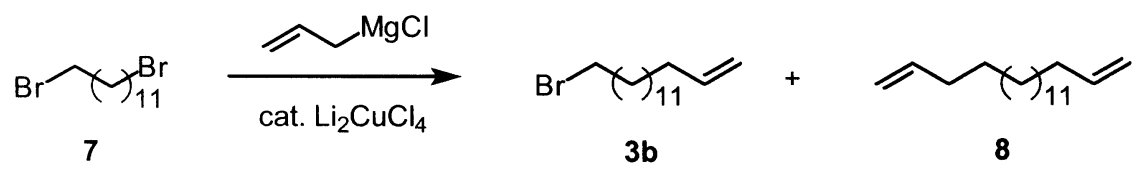

Scheme 3. Cu-catalyzed cross-coupling reaction to yield a $\omega$-bromo- $\alpha$-olefin, reagents, conditions, and yields: $\mathrm{LiCl}(0.4$ equiv. $)$, $\mathrm{CuCl}{ }_{2}(0.2$ equiv. $)$, allyl$\mathrm{MgCl}$ (1.5 equiv.), $\mathrm{THF}, 0^{\circ} \mathrm{C}, 1 \mathrm{~h}, \mathbf{3 b}: 47 \%, 7: 24 \%$ recovered, 8: $12 \%$.

\section{Conclusion}

Iron(III) chloride hexahydrate is an efficient catalytic system for seven-membered carboannulation by intramolecular Michael reaction of a cyclic oxoester with a vinyl ketone moiety. The reaction conditions are mild and not basic, the separation of the product from the catalyst is simply accomplished by filtration through $\mathrm{SiO}_{2}$, the yield is high and the diastereoselectivity quantitatively trans. In contrast to this, the above mentioned method is not suitable for a macrocarboannulation reaction. The synthesis of substrates for an intramolecular Michael reaction was achieved in a straightforward manner by introduction of an $\omega$-alkenyl side chain by cuprate addition to an $\alpha, \beta$-unsaturated oxoester followed by stepwise allylic oxidation to the enone moiety.

\section{Experimental}

\section{General}

${ }^{1} \mathrm{H}$ NMR spectra were recorded with Bruker DRX 500 (500 MHz), AM $400(400 \mathrm{MHz})$ and AC $200(200 \mathrm{MHz})$. ${ }^{13} \mathrm{C}$ NMR spectra were recorded with Bruker DRX 500 $(125 \mathrm{MHz})$ and AC $200(50 \mathrm{MHz}) .{ }^{1} \mathrm{H}$ and ${ }^{13} \mathrm{C}$ resonances were assigned by DEPT, HMBC, HMQC and H,H-COSY experiments. MS spectra were obtained with a Varian MAT 711 and MAT 955Q (high resolution). IR spectra were recorded with a Nicolet Magna IR 750. Elemental analyses were obtained with an Analytik Jena Vario EL.

Column chromatography was accomplished with Merck silica gel (Type 60, 0.063-0.200 mm). All manipulations involving Grignard or cuprate reagents were carried out in flame dried glassware under an atmosphere of $\mathrm{N}_{2}$ and with absolute THF, which was freshly distilled from potassium. $\mathrm{CuCl}_{2}, \mathrm{CuI}$, and $\mathrm{LiCl}$ were dried in high vacuum, $\mathrm{Mg}$ was activated with $\mathrm{I}_{2}$, and $\mathrm{SeO}_{2}$ freshly sublimated prior to use. All other reagents were used as purchased. All starting materials were commercially available, except methyl 7-oxo-1-cycloheptene-1-carboxylate (4), which was obtained according to a literature protocol. ${ }^{30}$ Solutions of allyl magnesium chloride and tert-butyl hydroperoxide were purchased from the Aldrich Chemical Co.

15-Bromo-1-pentadecene (3b). Solutions of $\mathrm{LiCl}(672 \mathrm{mg}$, $15.8 \mathrm{mmol})$ and $\mathrm{CuCl}_{2}(1.07 \mathrm{~g}, 7.92 \mathrm{mmol})$ in abs. THF (each $30 \mathrm{ml}$ ) were combined and stirred over night at room temperature. 1,12-Dibromododecane (7) (13.0 g, $39.6 \mathrm{mmol}$ ) was added in one portion, and allyl magnesium chloride ( $60.0 \mathrm{mmol}, 30.0 \mathrm{ml}$ of a $2 \mathrm{~mol} / \mathrm{l}$ solution in THF) was added within $30 \mathrm{~min}$ at $0^{\circ} \mathrm{C}$, and the resulting mixture was stirred for $60 \mathrm{~min}$ at this temperature. Hydrochloric acid $(100 \mathrm{ml}, 1 \mathrm{~mol} / \mathrm{l})$ was added, and the mixture was stirred for
$30 \mathrm{~min}$ at room temperature. Then the layers were separated, and the aqueous layer extracted with MTB (three times $50 \mathrm{ml}$ ). The combined organic layers were washed with brine $(20 \mathrm{ml})$ and dried over $\mathrm{MgSO}_{4}$. After filtration and evaporation of the solvent the crude product mixture was separated by chromatography $\left(\mathrm{SiO}_{2}, \mathrm{PE}\right)$ to give three fractions: First, byproduct $\mathbf{8}$ (colorless wax, $R_{\mathrm{f}}=0.56,1.19 \mathrm{~g}, 4.75 \mathrm{mmol}, 12 \%$ ) was obtained. Secondly, title compound $\mathbf{3 b}$ was eluted (colorless oil, $R_{\mathrm{f}}=0.41$, $5.37 \mathrm{~g}, 18.6 \mathrm{mmol}, 47 \%)$. As the third fraction, starting material 7 was recovered $\left(R_{\mathrm{f}}=0.35,3.15 \mathrm{~g}, 9.60 \mathrm{mmol}\right.$, 24\%). ${ }^{1} \mathrm{H}$ NMR $\left(\mathrm{CDCl}_{3}, 400 \mathrm{MHz}\right): \delta=1.26-1.54(\mathrm{~m}$, $20 \mathrm{H}), 1.85$ (pentet, $\left.J=7.1 \mathrm{~Hz}, 2 \mathrm{H}, 14-\mathrm{CH}_{2}\right), 2.04$ (q, $\left.J=7.1 \mathrm{~Hz}, 2 \mathrm{H} ; 3-\mathrm{CH}_{2}\right), 3.40\left(\mathrm{t}, J=6.9 \mathrm{~Hz}, 2 \mathrm{H} ; 15-\mathrm{CH}_{2}\right)$, $4.93(\mathrm{~d}, J=10.2 \mathrm{~Hz}, 1 \mathrm{H} ; E-1-\mathrm{CH}), 5.01(\mathrm{dq}, J=17.1 \mathrm{~Hz}$, $J=1.7 \mathrm{~Hz}, \quad 1 \mathrm{H} ; \quad Z-1-\mathrm{CH}), \quad 5.82 \quad$ (ddt, $J=17.0 \mathrm{~Hz}$, $J=10.2 \mathrm{~Hz}, J=6.6 \mathrm{~Hz}, 1 \mathrm{H} ; 2-\mathrm{CH}) \mathrm{ppm} .{ }^{13} \mathrm{C}\left\{{ }^{1} \mathrm{H}\right\} \mathrm{NMR}$ $\left(\mathrm{CDCl}_{3}, 50 \mathrm{MHz}\right): \delta=28.18\left(\mathrm{CH}_{2}\right), 28.77\left(\mathrm{CH}_{2}\right), 28.94$ $\left(\mathrm{CH}_{2}\right), 29.14\left(\mathrm{CH}_{2}\right), 29.44\left(\mathrm{CH}_{2}\right), 29.50\left(\mathrm{CH}_{2}\right), 29.53$ $\left(\mathrm{CH}_{2}\right), 29.59\left(\mathrm{CH}_{2}\right), 29.62\left(\mathrm{CH}_{2}\right), 29.69\left(\mathrm{CH}_{2}\right), 32.85$ $\left(\mathrm{CH}_{2}\right), 33.81\left(\mathrm{CH}_{2}\right), 33.87\left(\mathrm{CH}_{2}\right), 114.05\left(\mathrm{CH}_{2}\right), 139.16$ (CH) ppm. IR (ATR): 1/ $\lambda=2917$ (vs), 2851 (vs), 1641 (m), $1472(\mathrm{~m}), 1463(\mathrm{~m}), 1438(\mathrm{~m}), 992(\mathrm{~m}), 909$ (s), 717 (m) $\mathrm{cm}^{-1}$. MS (EI, $\left.70 \mathrm{eV}\right), \mathrm{m} / \mathrm{z}(\%): 288(2)\left[\mathrm{M}^{+}\right], 97$ (66), 83 (80), 69 (83), 55 (100) $\left[\mathrm{CH}_{2}=\mathrm{CH}-\left(\mathrm{CH}_{2}\right)_{2}^{+}\right] . \mathrm{C}_{15} \mathrm{H}_{29} \mathrm{Br}$ (289.30): Anal. calcd C 62.28, H 10.10; found C 62.30, H 10.46. Mol. mass calcd 288.1453, found $288.1457\left(\mathrm{M}^{+}\right.$, HRMS).

1,17-Octadecadiene (8). $R_{\mathrm{f}}\left(\mathrm{SiO}_{2}, \mathrm{PE}\right)=0.56 .{ }^{1} \mathrm{H} \quad \mathrm{NMR}$ $\left(\mathrm{CDCl}_{3}, 400 \mathrm{MHz}\right): \delta=1.17-1.38(\mathrm{~m}, 24 \mathrm{H}), 2.04$ (q, $\left.J=7.1 \mathrm{~Hz}, 4 \mathrm{H} ; 3-\mathrm{CH}_{2}, 16-\mathrm{CH}_{2}\right), 4.92(\mathrm{dq}, J=10.2 \mathrm{~Hz}$, $J=1.7 \mathrm{~Hz}, 2 \mathrm{H} ; E-1-\mathrm{CH}, E-18-\mathrm{CH}), 4.99(\mathrm{dq}, J=17.1 \mathrm{~Hz}$, $J=1.6 \mathrm{~Hz}, 2 \mathrm{H} ; Z-1-\mathrm{CH}, Z-18-\mathrm{CH}), 5.81$ (ddt, $J=17.0 \mathrm{~Hz}$, $J=10.3 \mathrm{~Hz}, J=6.8 \mathrm{~Hz}, 2 \mathrm{H} ; 2-\mathrm{CH}, 17-\mathrm{CH}) \operatorname{ppm} .{ }^{13} \mathrm{C}\left\{{ }^{1} \mathrm{H}\right\}$ NMR $\left(\mathrm{CDCl}_{3}, 50 \mathrm{MHz}\right): \delta=26.93\left(\mathrm{CH}_{2}\right), 28.97\left(\mathrm{CH}_{2}\right)$, $29.18\left(\mathrm{CH}_{2}\right), 29.53\left(\mathrm{CH}_{2}\right), 29.64\left(\mathrm{CH}_{2}\right), 29.72\left(\mathrm{CH}_{2}\right)$, $33.84\left(\mathrm{CH}_{2}\right), 114.06\left(\mathrm{CH}_{2}\right), 139.27(\mathrm{CH}) \mathrm{ppm}$. IR (ATR): $1 / \lambda=3077(\mathrm{~m}), 2977(\mathrm{~m}), 2923$ (vs), 2853 (s), 1641 (m), 1466 (m), 1440 (m), 992 (m), 909 (s), $721(\mathrm{~m}) \mathrm{cm}^{-1}$. MS (EI, $70 \mathrm{eV}), m / z(\%): 250$ (1) [ $\left.\mathrm{M}^{+}\right], 97$ (42), 83 (49), 69 (62), 55 (100) $\left[\mathrm{CH}_{2}=\mathrm{CH}-\left(\mathrm{CH}_{2}\right)_{2}^{+}\right] \cdot \mathrm{C}_{18} \mathrm{H}_{34}$ (250.46): Mol. mass calcd 250.2661, found 250.2663 (M+ $\left.\mathrm{M}^{+}, \mathrm{HRMS}\right)$.

Methyl 3-(4-penten-1-yl)cycloheptanone-2-carboxylate (5a). A Grignard solution prepared from 5-bromo-1-pentene (3a) $(1.77 \mathrm{~g}, 11.9 \mathrm{mmol})$ and $\mathrm{Mg}$ turnings (442 $\mathrm{mg}$, $18.2 \mathrm{mmol})$ in THF $(10 \mathrm{ml})$ was added at $-20^{\circ} \mathrm{C}$ to a suspension of $\mathrm{CuI}(1.82 \mathrm{~g}, 9.55 \mathrm{mmol})$ in $\mathrm{THF}(20 \mathrm{ml})$. After stirring for $1 \mathrm{~h}$ at $-20^{\circ} \mathrm{C}$ the mixture was cooled to $-78^{\circ} \mathrm{C}$, and a solution of acceptor $4(1.42 \mathrm{~g}, 8.42 \mathrm{mmol})$ in THF $(2 \mathrm{ml})$ was added. The mixture was stirred for $15 \mathrm{~min}$ at $-78^{\circ} \mathrm{C}$, then warmed up to room temperature within $1 \mathrm{~h}$. $\mathrm{NH}_{4} \mathrm{Cl}\left(50 \mathrm{ml}\right.$ of a saturated solution in $\left.\mathrm{H}_{2} \mathrm{O}\right)$ and conc. hydrochloric acid $(2 \mathrm{ml})$ were added, and the resulting 
suspension was extracted with MTB (three times $50 \mathrm{ml}$ ). After drying $\left(\mathrm{Na}_{2} \mathrm{SO}_{4}\right)$ and filtration the combined organic layers were evaporated and the residue chromatographed on $\mathrm{SiO}_{2}\left(\mathrm{PE} / \mathrm{MTB} 1: 1, R_{\mathrm{f}}=0.50\right)$ to yield the compound $\mathbf{5 a}$ as a colorless oil (1.70 g, $7.13 \mathrm{mmol}, 85 \%) .{ }^{1} \mathrm{H} \mathrm{NMR}\left(\mathrm{CDCl}_{3}\right.$, $400 \mathrm{MHz}$ ), a mixture of two diastereoisomers, trans/ cis $=2: 1$, trans: $\delta=1.73-2.08(\mathrm{~m}, 12 \mathrm{H}), 2.18-2.24(\mathrm{~m}$, $1 \mathrm{H}), 2.36-2.43(\mathrm{~m}, 1 \mathrm{H}), 2.63-2.82(\mathrm{~m}, 1 \mathrm{H}), 3.27(\mathrm{~d}$, $J=9.6 \mathrm{~Hz}, 1 \mathrm{H} ; 2-\mathrm{CH}), 3.71(\mathrm{~s}, 3 \mathrm{H} ; \mathrm{OMe}), 4.92-5.01(\mathrm{~m}$, $\left.2 \mathrm{H} ; 5^{\prime}-\mathrm{CH}_{2}\right), 5.71-5.83\left(\mathrm{~m}, 1 \mathrm{H} ; 4^{\prime}-\mathrm{CH}\right) \mathrm{ppm}$; cis: $\delta=1.73-$ $2.08(\mathrm{~m}, 13 \mathrm{H}), 2.44-2.50(\mathrm{~m}, 1 \mathrm{H}), 2.55-2.61(\mathrm{~m}, 1 \mathrm{H}), 3.71$ (s, 3H; OMe), 3.73 (d, J=6.3 Hz, 1H; 2-CH), 4.92-5.01 (m, $\left.2 \mathrm{H} ; 5^{\prime}-\mathrm{CH}_{2}\right), 5.71-5.83\left(\mathrm{~m}, 1 \mathrm{H} ; 4^{\prime}-\mathrm{CH}\right) \mathrm{ppm} .{ }^{13} \mathrm{C}\left\{{ }^{1} \mathrm{H}\right\}$ NMR $\left(\mathrm{CDCl}_{3}, 50 \mathrm{MHz}\right)$, a mixture of two diastereoisomers, trans (major): $\delta=25.74\left(\mathrm{CH}_{2}\right), 25.89\left(\mathrm{CH}_{2}\right), 27.79\left(\mathrm{CH}_{2}\right)$, $31.71\left(\mathrm{CH}_{2}\right), 33.63\left(\mathrm{CH}_{2}\right), 34.26\left(\mathrm{CH}_{2}\right), 37.81(\mathrm{CH}), 41.88$ $\left(\mathrm{CH}_{2}\right), 52.32\left(\mathrm{CH}_{2}\right), 65.89\left(\mathrm{CH}_{3}\right), 114.68\left(\mathrm{CH}_{2}\right), 138.40$ $(\mathrm{CH}), 170.21(\mathrm{C}=\mathrm{O}), 208.05(\mathrm{C}=\mathrm{O}) \mathrm{ppm}$; cis (minor): $\delta=24.03\left(\mathrm{CH}_{2}\right), 26.65\left(\mathrm{CH}_{2}\right), 26.88\left(\mathrm{CH}_{2}\right), 27.03\left(\mathrm{CH}_{2}\right)$, $31.24\left(\mathrm{CH}_{2}\right), 32.00\left(\mathrm{CH}_{2}\right), 37.33(\mathrm{CH}), 43.30\left(\mathrm{CH}_{2}\right), 51.77$ $(\mathrm{CH}), 62.34\left(\mathrm{CH}_{3}\right), 114.55\left(\mathrm{CH}_{2}\right), 138.56(\mathrm{CH}), 170.54$ $(\mathrm{C}=\mathrm{O}), 208.63(\mathrm{C}=\mathrm{O}) \mathrm{ppm}$. IR (ATR): $1 / \lambda=2930(\mathrm{~s})$, 2859 (m), 1744 (vs), 1707 (vs), 1640 (m), 1435 (s), 1303 (m), 1232 (s), 1200 (s), 1157 (s), 995 (m), $911(\mathrm{~m}) \mathrm{cm}^{-1}$. MS (EI, $70 \mathrm{eV}), m / z$ (\%): 238 (2) $\left[\mathrm{M}^{+}\right], 206$ (17), 169 (100) $\left[\mathrm{M}^{+}-\left(\mathrm{CH}_{2}\right)_{3} \mathrm{CH}=\mathrm{CH}_{2}\right], 137$ (44). $\mathrm{C}_{14} \mathrm{H}_{22} \mathrm{O}_{3}$ (238.33): Mol. mass calcd 238.1569, found 238.1567 (HRMS).

Methyl 3-(14-pentadecen-1-yl)cycloheptanone-2-carboxylate (5b). A Grignard solution prepared from $3 \mathbf{b}(2.54 \mathrm{~g}$, $8.77 \mathrm{mmol}$ ) and activated $\mathrm{Mg}$ turnings (320 mg, $13.2 \mathrm{mmol})$ in THF $(20 \mathrm{ml}$, ultrasonication was required to start the reaction) was added at $-30^{\circ} \mathrm{C}$ to a suspension of $\mathrm{CuI}(1.31 \mathrm{~g}, 6.89 \mathrm{mmol})$ in THF $(20 \mathrm{ml})$. After stirring for $2 \mathrm{~h}$ at $-15^{\circ} \mathrm{C}$ the mixture was cooled to $-78^{\circ} \mathrm{C}$, and a solution of acceptor 4 (1.05 g, $6.27 \mathrm{mmol})$ in THF $(10 \mathrm{ml})$ was added. The mixture was stirred for $15 \mathrm{~min}$ at $-78^{\circ} \mathrm{C}$, then warmed up to room temperature within $1 \mathrm{~h} . \mathrm{NH}_{4} \mathrm{Cl}$ ( $300 \mathrm{ml}$ of a saturated solution in $\mathrm{H}_{2} \mathrm{O}$ ) was added, and the resulting suspension was filtered through a glass frit. The aqueous layer was extracted with MTB (three times $100 \mathrm{ml})$. After drying $\left(\mathrm{Na}_{2} \mathrm{SO}_{4}\right)$ and filtration the combined organic layers were evaporated and the residue chromatographed twice on $\mathrm{SiO}_{2}$ (1. PE/MTB 2: $1, R_{\mathrm{f}}=0.50 ; 2$. PE/MTB $10: 1, R_{\mathrm{f}}=0.16$ ) to yield the compound $\mathbf{5 b}$ as a colorless oil (845 mg, $2.23 \mathrm{mmol}, 36 \%) .{ }^{1} \mathrm{H} \mathrm{NMR}\left(\mathrm{CDCl}_{3}\right.$, $400 \mathrm{MHz}$ ), trans-isomer (the cis-isomer is less than 5\%): $\delta=1.09-1.89\left(\mathrm{~m}, 30 \mathrm{H} ; 15 \mathrm{CH}_{2}\right), 2.01(\mathrm{q}, J=7.0 \mathrm{~Hz}, 2 \mathrm{H}$; $\left.13^{\prime}-\mathrm{CH}_{2}\right), 2.18-2.47\left(\mathrm{~m}, 3 \mathrm{H} ; 7-\mathrm{CH}_{2}, 3-\mathrm{CH}\right), 3.26$ (d, $J=9.5 \mathrm{~Hz}, 1 \mathrm{H} ; 2-\mathrm{CH}), 3.70$ (s, 3H; OMe), 4.90 (d, $\left.J=10.1 \mathrm{~Hz}, \quad 1 \mathrm{H} ; \quad 15^{\prime}-E-\mathrm{CHH}\right), 4.97 \quad(\mathrm{dd}, \quad J=17.0 \mathrm{~Hz}$, $\left.J=1.8 \mathrm{~Hz}, \quad 1 \mathrm{H} ; \quad 15^{\prime}-Z-\mathrm{CH} H\right), \quad 5.79 \quad(\mathrm{ddt}, \quad J=17.0 \mathrm{~Hz}$, $\left.J=10.2 \mathrm{~Hz}, J=6.7 \mathrm{~Hz}, 1 \mathrm{H} ; 14^{\prime}-\mathrm{CH}\right) \mathrm{ppm} .{ }^{13} \mathrm{C}\left\{{ }^{1} \mathrm{H}\right\} \mathrm{NMR}$ $\left(\mathrm{CDCl}_{3}, 50 \mathrm{MHz}\right)$, a mixture of two diastereoisomers, trans (major): $\delta=23.97\left(\mathrm{CH}_{2}\right), 25.76\left(\mathrm{CH}_{2}\right), 26.33\left(\mathrm{CH}_{2}\right)$, $27.54\left(\mathrm{CH}_{2}\right), 27.68\left(\mathrm{CH}_{2}\right), 28.80\left(\mathrm{CH}_{2}\right), 29.00\left(\mathrm{CH}_{2}\right), 29.37$ $\left(\mathrm{CH}_{2}\right), 29.50$ (br., $\left.5 \mathrm{CH}_{2}\right), 31.61\left(\mathrm{CH}_{2}\right), 33.66\left(\mathrm{CH}_{2}\right), 34.70$ $\left(\mathrm{CH}_{2}\right), 37.77$ (3-CH), $41.70\left(\mathrm{CH}_{2}\right), 52.01(\mathrm{OMe}), 65.75$ $(2-\mathrm{CH}), \quad 113.91 \quad\left(15^{\prime}-\mathrm{CH}_{2}\right), 138.90 \quad\left(14^{\prime}-\mathrm{CH}\right), \quad 170.03$ $(\mathrm{C}=\mathrm{O}), 207.63(\mathrm{C}=\mathrm{O}) \mathrm{ppm}$; cis-isomer (missing signals are hidden by the major isomer): $\delta=22.53\left(\mathrm{CH}_{2}\right), 24.51$ $\left(\mathrm{CH}_{2}\right), 24.78\left(\mathrm{CH}_{2}\right), 26.67\left(\mathrm{CH}_{2}\right), 26.75\left(\mathrm{CH}_{2}\right), 29.71$ $\left(\mathrm{CH}_{2}\right), 31.72\left(\mathrm{CH}_{2}\right), 31.76\left(\mathrm{CH}_{2}\right), 31.87\left(\mathrm{CH}_{2}\right), 34.99$
$\left(\mathrm{CH}_{2}\right), 43.10\left(\mathrm{CH}_{2}\right), 37.33(3-\mathrm{CH}), 51.47(\mathrm{OMe}), 62.22$ $(2-\mathrm{CH}), \quad 113.91 \quad\left(15^{\prime}-\mathrm{CH}_{2}\right), \quad 138.90 \quad\left(14^{\prime}-\mathrm{CH}\right), \quad 170.32$ $(\mathrm{C}=\mathrm{O}), 208.23(\mathrm{C}=\mathrm{O}) \mathrm{ppm}$. IR (ATR): $1 / \lambda=2923$ (vs), 2852 (vs), 1746 (s), 1708 (vs), 1640 (m), 1462 (m), 1435 (m), 1230 (m), 1198 (m), 1158 (m), 994 (m), 908 (m), 720 (m) $\mathrm{cm}^{-1}$. MS (EI, $\left.70 \mathrm{eV}\right), \mathrm{m} / \mathrm{z}(\%): 378$ (1) $\left[\mathrm{M}^{+}\right], 169$ (92), 137 (46), 111, 97 (50), 83 (85), 69 (63), 55 (100) $\left[\mathrm{CH}_{2}=\mathrm{CH}\left(\mathrm{CH}_{2}\right)_{2}^{+}\right] . \quad \mathrm{C}_{24} \mathrm{H}_{42} \mathrm{O}_{3} \quad$ (378.59): Anal. calcd C 76.14, H 11.18; found C 76.04, H 11.33. Mol. mass calcd 378.3134, found 378.3134 ( $\mathrm{M}^{+}$, HRMS).

Methyl 3-(3-hydroxy-4-penten-1-yl)cycloheptanone-2carboxylate (6a). Oxoester 5a $(1.50 \mathrm{~g}, 6.30 \mathrm{mmol}), \mathrm{SeO}_{2}$ $(1.36 \mathrm{~g}, 12.6 \mathrm{mmol}), \quad t \mathrm{BuO}_{2} \mathrm{H}(33 \mathrm{mmol}, 5.5 \mathrm{ml}$ of a $6.0 \mathrm{~mol} / \mathrm{l}$ solution in decane) and $\mathrm{CH}_{2} \mathrm{Cl}_{2}(10 \mathrm{ml})$ were mixed and stirred for $15 \mathrm{~h}$ at room temperature. The supernatant was decanted, the residue washed twice with $\mathrm{CH}_{2} \mathrm{Cl}_{2}$ (after drying, this material could be resubmitted to $\mathrm{SeO}_{2}$ sublimation), and from the combined $\mathrm{CH}_{2} \mathrm{Cl}_{2}$ solutions all volatile materials were removed in vacuo. The resulting crude oil was chromatographed on $\mathrm{SiO}_{2}$ (PE/MTB 1:1) to yield three fractions: First, starting material 5a was recovered (colorless oil, $R_{\mathrm{f}}=0.50,789 \mathrm{mg}$ of a mixture with $t \mathrm{BuO}_{2} \mathrm{H}$, molar ratio $1: 2.5$ by ${ }^{1} \mathrm{H}$ NMR, $1.70 \mathrm{mmol}$, $27 \%$; this mixture was directly resubmitted to another $\mathrm{SeO}_{2}$ oxidation). Secondly, enone 1a was yielded (colorless oil, $\left.R_{\mathrm{f}}=0.17,476 \mathrm{mg}, 1.89 \mathrm{mmol}, 30 \%\right)$. And finally, allylic alcohol 6a was obtained as a colorless oil $\left(R_{\mathrm{f}}=0.12,608 \mathrm{mg}\right.$, $2.39 \mathrm{mmol}, 38 \%)$. ${ }^{1} \mathrm{H} \mathrm{NMR}\left(\mathrm{CDCl}_{3}, 400 \mathrm{MHz}\right)$, a mixture of four diastereoisomers: $\delta=1.24-2.77(\mathrm{~m}, 13 \mathrm{H}), 3.13-$ $3.32(\mathrm{~m}, 1 \mathrm{H} ; 3-\mathrm{CH}), 3.71(\mathrm{~s}, 3 \mathrm{H}$; OMe), 3.66-3.82 (m, $1 \mathrm{H} ; 2-\mathrm{CH}), 4.04-4.11\left(\mathrm{~m}, 1 \mathrm{H} ; 3^{\prime}-\mathrm{CH}\right), 5.07-5.11(\mathrm{~m}, 1 \mathrm{H}$; $\left.5^{\prime}-\mathrm{CHH}\right), 5.18-5.24\left(\mathrm{~m}, 1 \mathrm{H} ; 5^{\prime}-\mathrm{CH}\right), 5.77-5.88(\mathrm{~m}, 1 \mathrm{H}$; $\left.4^{\prime}-\mathrm{CH}\right) \mathrm{ppm} .{ }^{13} \mathrm{C}\left\{{ }^{1} \mathrm{H}\right\} \mathrm{NMR}\left(\mathrm{CDCl}_{3}, 50 \mathrm{MHz}\right)$, a mixture of four diastereoisomers, two major and two minor isomers, major isomers: $\delta=25.62\left(2 \mathrm{CH}_{2}\right), 27.45\left(2 \mathrm{CH}_{2}\right), 30.17$ $\left(\mathrm{CH}_{2}\right), 30.28\left(\mathrm{CH}_{2}\right), 31.44\left(\mathrm{CH}_{2}\right), 31.57\left(\mathrm{CH}_{2}\right), 33.54$ $\left(\mathrm{CH}_{2}\right), 33.59\left(\mathrm{CH}_{2}\right), 37.38(\mathrm{CH}), 37.49(\mathrm{CH}), 41.72(2$ $\left.\mathrm{CH}_{2}\right), 52.21(\mathrm{CH}), 52.24(\mathrm{CH}), 65.45\left(\mathrm{CH}_{3}\right), 65.48\left(\mathrm{CH}_{3}\right)$, $72.36(\mathrm{CH}), 72.76(\mathrm{CH}), 114.43\left(\mathrm{CH}_{2}\right), 114.68\left(\mathrm{CH}_{2}\right)$, $140.75(\mathrm{CH}), \quad 140.84(\mathrm{CH}), \quad 170.04 \quad(\mathrm{C}=\mathrm{O}), \quad 170.12$ $(\mathrm{C}=\mathrm{O}), \quad 207.79 \quad(\mathrm{C}=\mathrm{O}), 207.86 \quad(\mathrm{C}=\mathrm{O})$ ppm; minor isomers: $\delta=23.72\left(\mathrm{CH}_{2}\right), 23.79\left(\mathrm{CH}_{2}\right), 24.62\left(\mathrm{CH}_{2}\right), 25.98$ $\left(\mathrm{CH}_{2}\right), 26.31\left(\mathrm{CH}_{2}\right), 26.61\left(\mathrm{CH}_{2}\right), 31.84\left(\mathrm{CH}_{2}\right), 34.70\left(\mathrm{CH}_{2}\right)$, $34.84\left(\mathrm{CH}_{2}\right), 34.97\left(\mathrm{CH}_{2}\right), 36.49(\mathrm{CH}), 37.11(\mathrm{CH}), 43.13$ $\left(\mathrm{CH}_{2}\right), 43.18\left(\mathrm{CH}_{2}\right), 51.69(\mathrm{CH}), 51.73(\mathrm{CH}), 61.87\left(\mathrm{CH}_{3}\right)$, $62.05\left(\mathrm{CH}_{3}\right), 72.04(2 \mathrm{CH}), 114.29\left(\mathrm{CH}_{2}\right), 114.54\left(\mathrm{CH}_{2}\right)$, 141.10 (2 CH), $170.69(\mathrm{C}=\mathrm{O}), 174.09(\mathrm{C}=\mathrm{O}), 208.41$ $(\mathrm{C}=\mathrm{O}), 208.46(\mathrm{C}=\mathrm{O}) \mathrm{ppm}$. IR (ATR): $1 / \lambda=3460$ (s), 2934 (s), 2861 (m), 1738 (vs), 1703 (vs), 1435 (m), 1349 (m), 1318 (m), 1265 (m), 1227 (m), 1199 (s), 1158 (s), 1057 (m), 993 (m), 923 (m) $\mathrm{cm}^{-1}$. MS (EI, $\left.70 \mathrm{eV}\right), \mathrm{m} / \mathrm{z}(\%): 253$ (1) $\left[\mathrm{M}^{+}-\mathrm{H}\right], 237 \quad(10), 219 \quad(17), 205 \quad$ (100) [M $\left.\mathrm{M}^{+}-\mathrm{OH}-\mathrm{MeOH}\right], 137$ (80). $\mathrm{C}_{14} \mathrm{H}_{22} \mathrm{O}_{4}$ (254.33): Mol. mass calcd 253.1439 (for $\mathrm{C}_{14} \mathrm{H}_{21} \mathrm{O}_{4}$ ), found 253.1436 $\left(\mathrm{M}^{+}-\mathrm{H}, \mathrm{HRMS}\right)$.

Methyl 3-(13-hydroxy-14-pentadecen-1-yl)cycloheptanone-2-carboxylate (6b). A mixture of oxoester $\mathbf{5 b}$ (766 mg, $2.02 \mathrm{mmol}), \mathrm{SeO}_{2}(460 \mathrm{mg}, 4.14 \mathrm{mmol})$ and $t \mathrm{BuO}_{2} \mathrm{H}$ (12 mmol, $2.0 \mathrm{ml}$ of a $6.0 \mathrm{~mol} / \mathrm{l}$ solution in decane) was stirred for $16 \mathrm{~h}$ at ambient temperature. After filtration and evaporation of volatile materials the filtrate was 
chromatographed on $\mathrm{SiO}_{2}\left(\mathrm{PE} / \mathrm{MTB} 1: 1, R_{\mathrm{f}}=0.38\right)$ to yield the title compound $\mathbf{6 b}(481 \mathrm{mg}, 1.21 \mathrm{mmol}, 60 \%)$ as a colorless oil. ${ }^{1} \mathrm{H} \mathrm{NMR}\left(\mathrm{CDCl}_{3}, 400 \mathrm{MHz}\right)$, only one signal set, minor diastereoisomer not detectable: $\delta=1.15-1.30(\mathrm{~m}$, $21 \mathrm{H}), 1.45-1.65(\mathrm{~m}, 7 \mathrm{H}), 1.72-1.90(\mathrm{~m}, 3 \mathrm{H}), 2.15-2.25$ $(\mathrm{m}, 1 \mathrm{H}), 2.35-2.45(\mathrm{~m}, 1 \mathrm{H}), 2.70-2.83(\mathrm{~m}, 1 \mathrm{H}), 3.27(\mathrm{~d}$, $J=9.5 \mathrm{~Hz}, 1 \mathrm{H} ; 2-\mathrm{CH}), 3.71$ (s, 3H; OMe), 4.08 (q, $\left.J=6.3 \mathrm{~Hz}, 1 \mathrm{H} ; 13^{\prime}-\mathrm{CH}\right), 5.08(\mathrm{dt}, J=10.4 \mathrm{~Hz}, J=1.1 \mathrm{~Hz}$, $\left.1 \mathrm{H} ; \quad E-15^{\prime}-\mathrm{CH}\right), 5.20 \quad(\mathrm{dt}, J=17.3 \mathrm{~Hz}, J=1.1 \mathrm{~Hz}, 1 \mathrm{H}$; $Z-15^{\prime}-\mathrm{CH}$ ), 5.85 (ddd, $J=16.8 \mathrm{~Hz}, J=10.4 \mathrm{~Hz}, J=6.2 \mathrm{~Hz}$, $\left.1 \mathrm{H} ; 14^{\prime}-\mathrm{CH}\right)$ ppm. ${ }^{13} \mathrm{C}\left\{{ }^{1} \mathrm{H}\right\}$ NMR $\left(\mathrm{CDCl}_{3}, 50 \mathrm{MHz}\right)$, a mixture of two diastereoisomers, major: $\delta=25.30\left(\mathrm{CH}_{2}\right)$, $25.92\left(\mathrm{CH}_{2}\right), 26.47\left(\mathrm{CH}_{2}\right), 27.81\left(\mathrm{CH}_{2}\right), 29.43\left(\mathrm{CH}_{2}\right)$, $29.49\left(\mathrm{CH}_{2}\right), 29.57\left(\mathrm{CH}_{2}\right), 29.62\left(\mathrm{CH}_{2}\right), 31.71\left(\mathrm{CH}_{2}\right)$, $34.82\left(\mathrm{CH}_{2}\right), 37.04\left(\mathrm{CH}_{2}\right), 37.93(\mathrm{CH}), 41.89\left(\mathrm{CH}_{2}\right), 52.29$ $(\mathrm{CH}), 65.97\left(\mathrm{CH}_{3}\right), 73.24(\mathrm{CH}), 114.45\left(\mathrm{CH}_{2}\right), 141.34(\mathrm{CH})$, $170.31(\mathrm{C}=\mathrm{O}), 208.22(\mathrm{C}=\mathrm{O}) \mathrm{ppm}$; minor isomer (missing signals are hidden by the major isomer): $\delta=26.76\left(\mathrm{CH}_{2}\right)$, $27.68\left(\mathrm{CH}_{2}\right), 31.82\left(\mathrm{CH}_{2}\right), 31.99\left(\mathrm{CH}_{2}\right), 37.45(\mathrm{CH}), 43.30$ $\left(\mathrm{CH}_{2}\right), 51.76(\mathrm{CH}), 62.44\left(\mathrm{CH}_{3}\right), 170.60(\mathrm{C}=\mathrm{O}), 208.42$ $(\mathrm{C}=\mathrm{O}) \mathrm{ppm}$. IR (ATR): $1 / \lambda=3446$ (br, m), 2925 (vs), 2853 (vs), 1743 (s), 1706 (s), 1457 (m), 1435 (m), 1318 (m), $1262(\mathrm{~m}), 1231(\mathrm{~m}), 1198(\mathrm{~m}), 1158(\mathrm{~m}), 991(\mathrm{~m})$, $919(\mathrm{~m}), \mathrm{cm}^{-1}$. MS (EI, $\left.70 \mathrm{eV}\right), \mathrm{m} / \mathrm{z}(\%): 376$ (2)

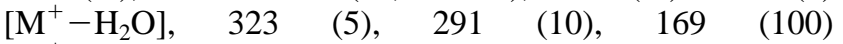
$\left[\mathrm{M}^{+}-\left(\mathrm{CH}_{2}\right)_{12} \mathrm{CH}(\mathrm{OH}) \mathrm{CH}=\mathrm{CH}_{2}\right], \quad 137$ (40), $111 \quad$ (30). $\mathrm{C}_{24} \mathrm{H}_{42} \mathrm{O}_{4}$ (394.59 g/mol): Mol. mass calcd 376.2977 (for $\left.\mathrm{C}_{24} \mathrm{H}_{40} \mathrm{O}_{3}\right)$, found $376.2973\left(\mathrm{M}^{+}-\mathrm{H}_{2} \mathrm{O}\right.$, HRMS $)$.

Methyl 3-(3-oxo-4-penten-1-yl)cycloheptanone-2-carboxylate (1a). A mixture of the allylic alcohol $6 \mathbf{a}(500 \mathrm{mg}$, $1.98 \mathrm{mmol}), \quad$ PCC $(641 \mathrm{mg}, 2.97 \mathrm{mmol})$ and $\mathrm{CH}_{2} \mathrm{Cl}_{2}$ $(10 \mathrm{ml})$ was stirred for $4 \mathrm{~h}$ at room temperature, then hydrochloric acid was added $(20 \mathrm{ml}, 1 \mathrm{~mol} / \mathrm{l})$. The liquid layers were decanted, and the residue dissolved in hydrochloric acid $(2 \mathrm{ml}, 6 \mathrm{~mol} / \mathrm{l})$. The combined aqueous layers were extracted with $\mathrm{CH}_{2} \mathrm{Cl}_{2}(2 \times 10 \mathrm{ml})$, and finally, the combined organic layers were filtered through a $\mathrm{SiO}_{2}$ column $(10 \mathrm{~cm}$, elution with MTB), which yielded compound 1a (400 mg, $1.58 \mathrm{mmol}, 80 \%$ ) as a colorless oil pure by TLC (PE/MTB $\left.1: 5, R_{\mathrm{f}}=0.43\right)$ and ${ }^{1} \mathrm{H}$ NMR. ${ }^{1} \mathrm{H}$ NMR $\left(\mathrm{CDCl}_{3}, 400 \mathrm{MHz}\right)$, a mixture of two diastereoisomers, trans/cis $=2: 1$, trans: $\delta=1.51-1.94\left(\mathrm{~m}, 8 \mathrm{H} ; 4 \mathrm{CH}_{2}\right), 2.22-2.78(\mathrm{~m}, 5 \mathrm{H} ; 3-\mathrm{CH}, 2$ $\left.\mathrm{CH}_{2}\right), 3.31(\mathrm{~d}, J=9.5 \mathrm{~Hz}, 1 \mathrm{H} ; 2-\mathrm{CH}), 3.71$ (s, 3H; OMe), $5.83\left(\mathrm{~d}, J=10.4 \mathrm{~Hz}, 1 \mathrm{H} ; E^{-} 5^{\prime}-\mathrm{CH} H\right), 6.21$ (dd, $J=17.6 \mathrm{~Hz}$, $\left.J=0.9 \mathrm{~Hz}, \quad 1 \mathrm{H} ; \quad Z-5^{\prime}-\mathrm{CHH}\right), \quad 6.34 \quad(\mathrm{dd}, \quad J=17.7 \mathrm{~Hz}$, $\left.J=10.4 \mathrm{~Hz}, 1 \mathrm{H} ; 4^{\prime}-\mathrm{CH}\right) \mathrm{ppm}$; cis: $\delta=1.51-1.94(\mathrm{~m}, 8 \mathrm{H} ; 4$ $\mathrm{CH}_{2}$ ), 2.22-2.78 (m, 5H; 3-CH, $2 \mathrm{CH}_{2}$ ), 3.71 (s, 3H; OMe), $3.72(\mathrm{~d}, J=4.1 \mathrm{~Hz}, 1 \mathrm{H}$; 2-CH), $5.83(\mathrm{~d}, J=10.4 \mathrm{~Hz}, 1 \mathrm{H}$; $\left.E-5^{\prime}-\mathrm{CH} H\right), 6.22\left(\mathrm{dd}, J=17.7 \mathrm{~Hz}, J=1.0 \mathrm{~Hz}, 1 \mathrm{H} ; Z_{-} 5^{\prime}-\right.$ $\mathrm{CHH}), 6.35$ (dd, $\left.J=17.7 \mathrm{~Hz}, J=10.5 \mathrm{~Hz}, 1 \mathrm{H} ; 4^{\prime}-\mathrm{CH}\right) \mathrm{ppm}$. ${ }^{13} \mathrm{C}\left\{{ }^{1} \mathrm{H}\right\}$ NMR $\left(\mathrm{CDCl}_{3}, 50 \mathrm{MHz}\right)$, a mixture of two diastereoisomers, trans (major): $\delta=25.70 \quad\left(\mathrm{CH}_{2}\right), 27.52$ $\left(\mathrm{CH}_{2}\right), 28.61\left(\mathrm{CH}_{2}\right), 31.69\left(\mathrm{CH}_{2}\right), 36.63\left(\mathrm{CH}_{2}\right), 37.35$ $(\mathrm{CH}), 41.87\left(\mathrm{CH}_{2}\right), 52.45\left(\mathrm{CH}_{3}\right), 65.38(\mathrm{CH}), 128.23$ $\left(5^{\prime}-\mathrm{CH}_{2}\right), 136.32\left(4^{\prime}-\mathrm{CH}\right), 169.98(\mathrm{C}=\mathrm{O}), 199.81(\mathrm{C}=\mathrm{O})$, $207.60(\mathrm{C}=\mathrm{O}) \mathrm{ppm}$; cis (minor): $\delta=23.93\left(\mathrm{CH}_{2}\right), 26.36$ $\left(\mathrm{CH}_{2}\right), 26.69\left(\mathrm{CH}_{2}\right), 32.29\left(\mathrm{CH}_{2}\right), 36.93(\mathrm{CH}), 37.76$ $\left(\mathrm{CH}_{2}\right), 43.25\left(\mathrm{CH}_{2}\right), 51.91\left(\mathrm{CH}_{3}\right), 62.04(\mathrm{CH}), 127.69$ $\left(5^{\prime}-\mathrm{CH}_{2}\right), 136.50\left(4^{\prime}-\mathrm{CH}\right), 170(\mathrm{C}=\mathrm{O}), 200.12(\mathrm{C}=\mathrm{O})$, $208.19(\mathrm{C}=\mathrm{O}) \mathrm{ppm}$. IR (ATR): $1 / \lambda=2932(\mathrm{~s}), 2862(\mathrm{~m})$, 1739 (s), 1704 (vs), 1616 (m), 1447 (m), 1436 (m), 1405 (m), 1352 (m), 1317 (m), 1264 (m), 1199 (s), 1156 (s), 1092 (m), $1017(\mathrm{~m}), 993(\mathrm{~m}) \mathrm{cm}^{-1}$. MS (EI, $\left.70 \mathrm{eV}\right), \mathrm{m} / \mathrm{z}(\%): 252$ (1) $\left[\mathrm{M}^{+}\right], 193$ (4), 182 (38), 169 (14), 150 (34), 137 (36), 55 (100) $\left[\mathrm{CH}_{2} \mathrm{CHCO}^{+}\right] . \mathrm{C}_{14} \mathrm{H}_{20} \mathrm{O}_{4}$ (252.31): Mol. mass calcd 252.1362, found $252.1367\left(\mathrm{M}^{+}, \mathrm{HRMS}\right)$.

Methyl 3-(13-oxo-14-pentadecen-1-yl)cycloheptanone-2carboxylate (1b). NMO (874 mg, $6.47 \mathrm{mmol}$ ) and ground molecular sieves ( $4 \AA, 828 \mathrm{mg}$ ) were added at ambient temperature to a solution of alcohol $\mathbf{6 b}$ (426 mg, $1.08 \mathrm{mmol})$ in abs. $\mathrm{CH}_{2} \mathrm{Cl}_{2}(10 \mathrm{ml})$. TPAP (60 mg, $0.16 \mathrm{mmol}$ ) was added and the mixture stirred for $1 \mathrm{~h}$ at $0^{\circ} \mathrm{C}$. After filtration through $\mathrm{SiO}_{2}$ (MTB) and evaporation, chromatography on $\mathrm{SiO}_{2}\left(\mathrm{PE} / \mathrm{MTB} 2: 1, R_{\mathrm{f}}=0.30\right)$ yielded the compound $\mathbf{1 b}$ (366 mg, $0.932 \mathrm{mmol}, 86 \%$ ) as a colorless oil. ${ }^{1} \mathrm{H} \mathrm{NMR}\left(\mathrm{CDCl}_{3}, 400 \mathrm{MHz}\right)$, only one signal set, minor diastereoisomer not detectable: $\delta=1.19-1.33(\mathrm{~m}, 13 \mathrm{H})$, $1.51-1.62(\mathrm{~m}, 8 \mathrm{H}), 1.74-2.04(\mathrm{~m}, 5 \mathrm{H}), 2.30-2.43(\mathrm{~m}$, $3 \mathrm{H}), 2.57\left(\mathrm{t}, J=7.4 \mathrm{~Hz}, 2 \mathrm{H} ; 12^{\prime}-\mathrm{CH}_{2}\right), 2.74-2.80(\mathrm{~m}, 2 \mathrm{H}$; 7- $\mathrm{CH}_{2}$ ), 3.27 (d, J=9.5 Hz, 1H; 2-CH), 3.72 (s, 3H; OMe), $5.81\left(\mathrm{dd}, J=10.4 \mathrm{~Hz}, J=0.9 \mathrm{~Hz}, 1 \mathrm{H} ; E-15^{\prime}-\mathrm{CH}\right), 6.21$ (dd, $\left.J=17.5 \mathrm{~Hz}, J=0.8 \mathrm{~Hz}, 1 \mathrm{H} ; Z-15^{\prime}-\mathrm{CH}\right), 6.35(\mathrm{dd}, J=17.7 \mathrm{~Hz}$, $\left.J=10.4 \mathrm{~Hz}, 1 \mathrm{H} ; 14^{\prime}-\mathrm{CH}\right)$ ppm. ${ }^{13} \mathrm{C}\left\{{ }^{1} \mathrm{H}\right\}$ NMR $\left(\mathrm{CDCl}_{3}\right.$, $50 \mathrm{MHz}$ ), a mixture of two diastereoisomers, major: $\delta=24.03\left(\mathrm{CH}_{2}\right), 25.34\left(\mathrm{CH}_{2}\right), 26.49\left(\mathrm{CH}_{2}\right), 27.83\left(\mathrm{CH}_{2}\right)$, $29.26\left(\mathrm{CH}_{2}\right), 29.41\left(\mathrm{CH}_{2}\right), 29.45\left(\mathrm{CH}_{2}\right), 29.57\left(\mathrm{CH}_{2}\right)$, $29.64\left(\mathrm{CH}_{2}\right), 31.73\left(\mathrm{CH}_{2}\right), 34.85\left(\mathrm{CH}_{2}\right), 39.67\left(\mathrm{CH}_{2}\right)$, $41.92\left(\mathrm{CH}_{2}\right), 52.30(\mathrm{CH}), 65.99\left(\mathrm{CH}_{3}\right), 127.80\left(\mathrm{CH}_{2}\right)$, $136.61(\mathrm{CH}), 170.30(\mathrm{C}=\mathrm{O}), 201.12(\mathrm{C}=\mathrm{O}), 208.21$ $(\mathrm{C}=\mathrm{O})$ ppm; minor isomer (missing signals are hidden by the major isomer): $\delta=24.11\left(\mathrm{CH}_{2}\right), 26.79\left(\mathrm{CH}_{2}\right), 26.91$ $\left(\mathrm{CH}_{2}\right), 27.70\left(\mathrm{CH}_{2}\right), 32.01\left(\mathrm{CH}_{2}\right), 43.32\left(\mathrm{CH}_{2}\right), 51.76$ $(\mathrm{CH}), 170.60(\mathrm{C}=\mathrm{O}), 208.78(\mathrm{C}=\mathrm{O}) \mathrm{ppm}$. IR (ATR): $1 / \lambda=2925$ (vs), 2853 (s), 1743 (s), 1706 (s), 1457 (m), 1435 (m), 1262 (m), 1231 (m), $1198(\mathrm{~m}), 1158$ (m), 991 (m), 919 (m) $\mathrm{cm}^{-1}$. MS (EI, $\left.70 \mathrm{eV}\right), \mathrm{m} / z(\%): 392(4)\left[\mathrm{M}^{+}\right], 361$ (18), 333 (12), $323 \quad$ (22), $291 \quad$ (38), $169 \quad$ (100) $\left[\mathrm{M}^{+}-\left(\mathrm{CH}_{2}\right)_{12} \mathrm{COCH}=\mathrm{CH}_{2}\right] . \quad \mathrm{C}_{24} \mathrm{H}_{40} \mathrm{O}_{4} \quad$ (392.58): Mol. mass calcd. 392.2927, found 392.2929 ( $\mathrm{M}^{+}$, HRMS).

trans-Methyl bicyclo[5.5.0]dodecan-2,11-dione-1-carboxylate (2a). Compound 1a (89 $\mathrm{mg}, 0.35 \mathrm{mmol})$ and $\mathrm{FeCl}_{3} \cdot 6 \mathrm{H}_{2} \mathrm{O}(4.7 \mathrm{mg}, 0.017 \mathrm{mmol})$ were stirred in $\mathrm{CH}_{2} \mathrm{Cl}_{2}$ $(1 \mathrm{ml})$ for $12 \mathrm{~h}$ at ambient temperature. Subsequently, the reaction mixture was transferred onto the top of a silica gel column, and the product 2a was eluted with PE/MTB (1:2, $\left.R_{\mathrm{f}}=0.33\right)$ to yield $71 \mathrm{mg}(80 \%)$ of $\mathbf{2 a}$ as a colorless oil. Also, an amount of about $10 \mathrm{mg}(11 \%)$ of the starting material $\mathbf{1 a}$ was recovered $\left(R_{\mathrm{f}}=0.38\right) .{ }^{1} \mathrm{H} \mathrm{NMR}\left(\mathrm{CDCl}_{3}, 500 \mathrm{MHz}\right)$ : $\delta=1.36(\mathrm{~m}, 1 \mathrm{H} ; 5-\mathrm{H}), 1.56(\mathrm{~m}, 1 \mathrm{H} ; 4-\mathrm{H}), 1.71(\mathrm{tt}$, $J=11 \mathrm{~Hz}, J=3 \mathrm{~Hz}, 1 \mathrm{H} ; 7-\mathrm{H}), 1.71-1.81 \quad(\mathrm{~m}, 2 \mathrm{H}$; 6-H, 8-H), 1.89-1.95 (m, 2H; 4-H, 5-H), 2.08-2.22 (m, 3H; 6-H, 8-H, 12-H), 2.27 (ddd, $J=15 \mathrm{~Hz}, J=6 \mathrm{~Hz}, J=3 \mathrm{~Hz}$, $1 \mathrm{H} ; 12-\mathrm{H}), 2.51$ (ddd, $J=16 \mathrm{~Hz}, J=6 \mathrm{~Hz}, J=3 \mathrm{~Hz}, 1 \mathrm{H}$; $11-\mathrm{H}), 2.54$ (ddd, $J=15 \mathrm{~Hz}, J=6 \mathrm{~Hz}, J=4 \mathrm{~Hz}, 1 \mathrm{H}$; 9-H), $2.57(\mathrm{~m}, 1 \mathrm{H} ; 3-\mathrm{H}), 2.61(\mathrm{~m}, 1 \mathrm{H} ; 9-\mathrm{H}), 2.65$ (ddd, $J=16 \mathrm{~Hz}, \quad J=12 \mathrm{~Hz}, \quad J=3 \mathrm{~Hz}, 1 \mathrm{H} ; 11-\mathrm{H}$ ), $2.70 \quad$ (ddd, $J=13 \mathrm{~Hz}, J=6 \mathrm{~Hz}, J=5 \mathrm{~Hz}, 1 \mathrm{H} ; 3-\mathrm{H}), 3.83$ (s, 3H; $\left.\mathrm{CH}_{3}\right)$ ppm. ${ }^{13} \mathrm{C}\left\{{ }^{1} \mathrm{H}\right\}$ NMR $\left(\mathrm{CDCl}_{3}, 50 \mathrm{MHz}\right): \delta=24.36(4-\mathrm{C})$, 28.44 (5-C), 29.51 (8-C), 31.20 (12-C), 36.17 (6-C), 39.47 (11-C), 41.49 (3-C), 42.57 (9-C), 46.11 (7-C), 52.02 (OMe), 66.14 (1-C), $171.84\left(\mathrm{CO}_{2} \mathrm{Me}\right), 209.68$ (2-C), 212.71 (10-C) ppm. IR (ATR): 1/ $\lambda=2935(\mathrm{~s}), 2862(\mathrm{~m}), 1735$ (vs), 1703 (vs), 1447 (s), 1449 (m), 1225 (s), 1158 (s), 1106 (m), 1019 
(m), $936(\mathrm{~m}), 799(\mathrm{~m}) \mathrm{cm}^{-1}$. MS (EI, $\left.70 \mathrm{eV}\right), \mathrm{m} / \mathrm{z}(\%): 252$ (27) $\left[\mathrm{M}^{+}\right], 234$ (10), 220 (16), 192 (26), 182 (52), 169 (46), 137 (100) [cyclo- $\left.\left(\mathrm{CH}_{2}\right)_{4} \mathrm{CHC}(\mathrm{CO}) \mathrm{CO}^{+}\right] . \quad \mathrm{C}_{14} \mathrm{H}_{20} \mathrm{O}_{4}$ (252.31): Mol. mass calcd 252.1362, found 252.1366 (HRMS).

Methyl bicyclo[15.5.0]docosan-4,22-dione-1-carboxylate (2b). Anhydrous $\mathrm{FeCl}_{3}(97.5 \mathrm{mg}, 0.0601 \mathrm{mmol})$ was added to a solution of oxoester $\mathbf{1 b}(236 \mathrm{mg}, 0.601 \mathrm{mmol})$ in abs. $\mathrm{CH}_{2} \mathrm{Cl}_{2}(6 \mathrm{ml})$. After stirring for $15 \mathrm{~min}$ a brown material precipitated. The mixture was kept for $2 \mathrm{~h}$ in an ultrasound bath resulting in a homogeneous dark brown solution, which was further stirred for $16 \mathrm{~h}$ at room temperature. Half conc. $\mathrm{HCl}(5 \mathrm{ml})$ was added, and the mixture extracted three times with $\mathrm{CH}_{2} \mathrm{Cl}_{2}$. The combined extracts were filtered through $\mathrm{SiO}_{2}\left(\mathrm{CH}_{2} \mathrm{Cl}_{2}\right)$ and evaporated. Twice chromatography on $\mathrm{SiO}_{2}\left(\mathrm{PE} / \mathrm{MTB} 2: 1, R_{\mathrm{f}}=0.24\right)$ yielded the macrocyclic product $\mathbf{2 b}(20 \mathrm{mg}, 0.051 \mathrm{mmol}, 8 \%)$ as a colorless material. IR (ATR): $1 / \lambda=2925$ (vs), 2853 (s), 1736 (s), 1707 (vs), 1447 (m), 1435 (m), 1365 (m), 1350 (m), 1319 (m), $1223(\mathrm{~m}), 1197(\mathrm{~m}), 1158(\mathrm{~m}) \mathrm{cm}^{-1}$. MS (EI, $\left.70 \mathrm{eV}\right)$, $\mathrm{m} / \mathrm{z}(\%): 392(8)\left[\mathrm{M}^{+}\right], 364(17), 360(24), 332$ (38), 304 (26), 277 (16), 169 (93), 137 (46), 109 (40), 95 (48), 81 (67), 69 (51), 67 (49), 57 (43), 55 (100). $\mathrm{C}_{24} \mathrm{H}_{40} \mathrm{O}_{4}$ (392.58): Mol. mass calcd 392.2927 (for $\mathrm{C}_{24} \mathrm{H}_{40} \mathrm{O}_{4}$ ), found $392.2928\left(\mathrm{M}^{+}\right.$, HRMS). ${ }^{1} \mathrm{H}$ NMR $\left(\mathrm{CDCl}_{3}, 500 \mathrm{MHz}\right)$ : very broad multiplets between 1.00 and $2.80 \mathrm{ppm}$, four signals to be assigned to $\mathrm{CO}_{2} \mathrm{Me}$ groups of four isomers: two diastereoisomers each having two conformers, $\delta=3.70,3.71,3.73,3.74 \mathrm{ppm}$. ${ }^{13} \mathrm{C}\left\{{ }^{1} \mathrm{H}\right\}$ NMR $\left(\mathrm{CDCl}_{3}, 125 \mathrm{MHz}\right)$ : very broad signals, due to bad signal to noise ratio no assignments possible.

\section{Acknowledgements}

This work was supported by the Fonds der Chemischen Industrie and the Deutsche Forschungsgemeinschaft. We are also grateful to Prof. S. Blechert and the Institut für Organische Chemie der Technischen Universität Berlin for support. J. C. also thanks the Deutsche Forschungsgemeinschaft and H. O. the Graduiertenkolleg 'Synthetische, mechanistische und reaktionstechnische Aspekte von Metallkatalysatoren' for fellowships.

\section{References}

1. Christoffers, J. Chem. Commun. 1997, 943-944.

2. Christoffers, J. J. Chem. Soc., Perkin Trans. 1 1997, 31413149 .

3. Review: Christoffers, J. Eur. J. Org. Chem. 1998, 1259-1266.

4. Review: Shibasaki, M.; Sasai, H.; Arai, T. Angew. Chem. 1997,
109, 1290-1311; Angew. Chem. Int. Ed. Engl. 1997, 36, 12361256.

5. Review: Perlmutter, P. Conjugate Addition Reactions in Organic Synthesis; Tetrahedron Organic Chemistry Series; Pergamon: Oxford, 1992; Vol. 9.

6. Review: Oare, D. A.; Heathcock, C. H. In Topics in Stereochemistry; Eliel, E. L.; Wilen, S. H. Eds.; Wiley Interscience: New York, 1989; Vol. 19, 227-407.

7. Review: Tomioka, K.; Koga, K. In Asymmetric Synthesis; Morrison J. D. Eds.; Academic Press: New York, 1983; Vol. 2, 201-224.

8. Review: Bergmann, E. D.; Ginsburg, D.; Pappo, R. Org. React. 1959, 10, 179-555.

9. Christoffers, J. Org. Synth. in press.

10. Ruel, R.; Deslongchamps, P. Can. J. Chem. 1990, 68, 19171922.

11. Lavallee, J.-F.; Deslongchamps, P. Tetrahedron Lett. 1987, 28, 3457-3458.

12. Berthiaume, G.; Lavallee, J.-F.; Deslongchamps, P. Tetrahedron Lett. 1986, 27, 5451-5454.

13. Lavallee, J.-F.; Berthiaume, G.; Deslongchamps, P. Tetrahedron Lett. 1986, 27, 5455-5458.

14. Krohn, K.; Müller, U.; Priyono, W.; Sarstedt, B.; Stoffregen, A. Liebigs Ann Chem. 1984, 306-318.

15. Review: Little, R. D.; Masjedizadeh, M. R.; Wallquist, O.; McLoughlin, J. I. Org. React. 1995, 47, 315-552.

16. Ullmann, A.; Reißig, H.-U.; Rademacher, O. Eur J. Org. Chem. 1998, 2541-2549.

17. Crevisy, C.; Couturier, M.; Dugave, C.; Dory, Y. L.; Deslongchamps, P. Bull. Chem. Soc. Fr. 1995, 132, 360-370.

18. Berthiaume, G.; Deslongchamps, P. Bull. Chem. Soc. Fr. 1995, 132, 371-383.

19. Deslongchamps, P.; Roy, B. L. Can J. Chem. 1986, 64, $2068-$ 2075.

20. Christoffers, J. Tetrahedron Lett. 1998, 39, 7083-7084.

21. Rossa, L.; Vögtle, F. Top. Curr. Chem. 1983, 113, 1-86.

22. Bruhn, J.; Heimgartner, H.; Schmid, H. Helv. Chim. Acta 1979, 62, 2630-2654.

23. Christoffers, J. J. Org. Chem. 1998, 63, 4539-4540.

24. Marshall, J. A.; Jenson, T. M.; DeHoff, B. S. J. Org. Chem. 1987, 52, 3860-3866.

25. Umbreit, M. A.; Sharpless, K. B. J. Am. Chem. Soc. 1977, 99 , 5526-5528.

26. Sharpless, K. B.; Lauer, R. F. J. Am. Chem. Soc. 1972, 94, 7154-7155.

27. Cainelli, G.; Cardillo, G. Chromium Oxidations in Organic Chemistry, Springer: Berlin, Heidelberg, 1984.

28. Ley, S. V.; Norman, J.; Griffith, W. P.; Marsden, S. P. Synthesis 1994, 639-666.

29. Johnson, D. K.; Donohoe, J.; Kang, J. Synth. Commun. 1994, 24, 1557-1564.

30. Christoffers, J. Eur J. Org. Chem. 1998, 759-761. 